\title{
Análise do potencial de Aquisição de dados magnetométricos por VANT
}

Coelho, D. C., IAG-USP; Shukowsky, W., IAG-USP.

Copyright 2018, SBGf - Sociedade Brasileira de Geofísica

Este texto foi preparado para a apresentação no VIII Simpósio Brasileiro de Geofísica, Salinópolis, 18 a 20 de setembro de 2018. Seu conteúdo foi revisado pelo Comitê Técnico do VIII SimBGf, mas não necessariamente representa a opinião da SBGf ou de seus associados. É proibida a reprodução total ou parcial deste material para propósitos comerciais sem prévia autorização da SBGf.

\section{Resumo}

Técnicas convencionais de magnetometria apresentam limitações relacionadas a custos e/ou acessibilidade da área a ser investigada. Neste sentido, explorar métodos mais eficientes torna-se relevante para a geofísica aplicada. Neste contexto, o objetivo deste trabalho foi avaliar o potencial de VANTs (Veículos Aéreos Não Tripulados), na aquisição de dados magnetométricos. Para tanto, desenvolveu-se um sistema de aquisição de dados magnetométricos leve e de baixo custo a partir de sensores microeletromecânicos, que foi aerotransportado por um VANT para a aquisição dos dados magnéticos. Anomalias magnéticas de uma área com alvos conhecidos foram detectadas. Os mapas gerados mostraram a anomalia prevista, evidenciando a eficácia da técnica. Em comparação com a magnetometria terrestre, o aerolevantamento por VANT apresentou uma qualidade de dados com menor resolução. Entretanto, sua capacidade de mapeamento, principalmente no que se refere a ambientes de difícil acesso, foi superior. Desta forma, de acordo com o objetivo do levantamento magnetométrico, o uso VANTs pode ser considerado mais eficiente. Adicionalmente, os custos associados ao aerolevantamento por VANT foram estimados em menos da metade do valor associado à magnetometria terrestre. Conclui-se, portanto, que os VANTs podem ser uma importante ferramenta para a geofísica aplicada.

\section{Introdução}

A magnetometria é uma técnica geofísica que usa as informações do campo geomagnético para a investigação das rochas e estruturas geológicas em subsuperfície. $\mathrm{O}$ campo magnético terrestre induz nos materiais geológicos um campo magnético secundário, que se soma ao campo principal e causa variações que podem ser medidas, através de instrumentos chamados "magnetômetros", e analisadas para a investigação das fontes causadoras dessas anomalias. A maioria das rochas existentes na crosta terrestre possui minerais capazes de produzir anomalias magnéticas. De maneira similar, alguns objetos feitos pelo homem a partir de materiais ferrosos também geram essas anomalias. Dessa forma, o levantamento magnético tem uma ampla variedade de aplicações, desde a engenharia e arqueologia, até levantamentos de grande escala para investigar estruturas geológicas regionais (KEAREY; BROOKS; HILL, 2009).

Os levantamentos magnéticos terrestres são geralmente realizados sobre áreas relativamente pequenas, sobre um alvo previamente definido. Consequentemente, o espaçamento entre as medidas é geralmente da ordem de 10 a 100 metros, embora espaçamentos menores possam ser empregados em situações específicas. Na aquisição de dados aeromagnéticos o espaçamento entre as linhas de voo depende do grau de detalhe exigido para o mapeamento ou alvo de exploração desejado, e está fortemente condicionado aos recursos financeiros disponíveis para o levantamento. Para levantamentos de reconhecimento são realizados espaçamentos da ordem de 1 a $2 \mathrm{~km}$, enquanto levantamentos de detalhe são voados com linhas espaçadas de 100 a 250 metros. Quanto à altura do voo (distância entre a plataforma aérea e o solo) esta deve ser a mais baixa possível de modo que não afete a segurança da operação. Nas últimas décadas, no Brasil, tem sido fixada em 100 metros para aeronaves de asa fixa e em 60 metros para levantamentos com helicóptero (MARANGONI, 2014).

Para exemplo de uma aplicação da magnetometria terrestre, no dia 12 de abril de 2011 ocorreu um acidente em uma pedreira, em Santos (SP), que resultou no soterramento de um caminhão e uma escavadeira e vitimou dois operadores após a ruptura de parte de um talude de cerca de 80 metros de altura e 120 metros de extensão (figura 1), em local onde havia um acesso para frente de exploração de brita (GALLI; CAMPOS; SOUSA, 2013).

Com o objetivo de auxiliar os integrantes do Corpo de Bombeiros e equipe de trabalhadores na busca dos operários, partindo do princípio que localizando o caminhão e a escavadeira possivelmente se encontrariam também os corpos das pessoas que as operavam, foi realizado um ensaio de magnetometria terrestre a fim de se delimitar as zonas magneticamente anômalas que pudessem ser associadas ao maquinário (GALLI; CAMPOS; SOUSA, 2013).

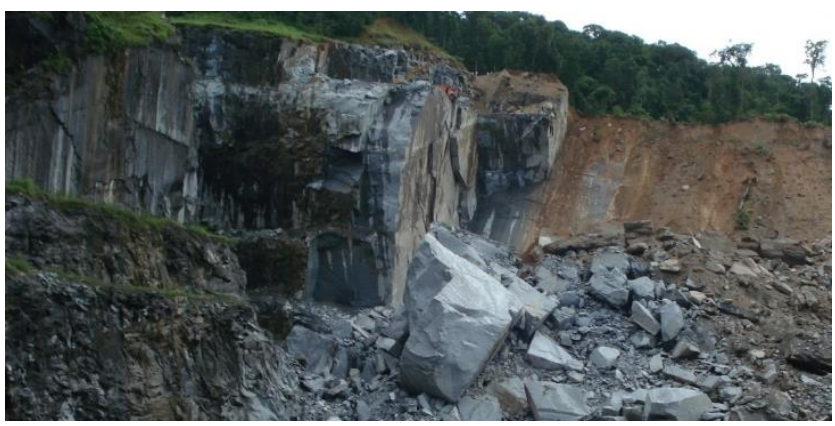

Figura 1 - Deslizamento de parte do talude que soterrou o maquinário. É possível notar alguns veículos no centro da imagem como fator de escala (GALLI; CAMPOS; SOUSA, 2013). 
Devido à grande dificuldade de acesso e a possibilidade de novos deslizamentos, a aquisição de dados ocorreu com a ajuda dos bombeiros, caminhando-se apenas pelas regiões mais seguras. Os pontos de aquisição de dados podem ser vistos na figura 2 (GALLI; CAMPOS; SOUSA, 2013).

Ao final do trabalho foi possível determinar a posição do maquinário soterrado. Com a remoção das rochas no local indicado, o maquinário e os corpos das vítimas foram localizados (GALLI; CAMPOS; SOUSA, 2013).

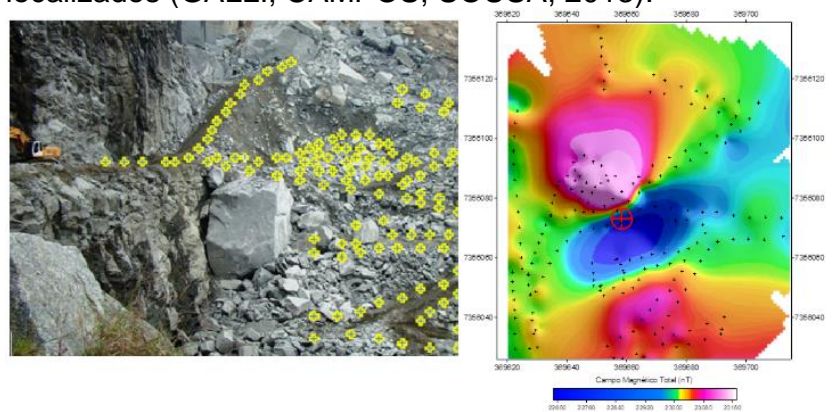

Figura 2 - Região da pedreira onde foram obtidos os dados magnetométricos (esquerda). Pontos amarelos indicam o trajeto e posição da tomada de cada dado com a ajuda dos bombeiros. A direita temos o mapa do dipolo magnético que representa os veículos soterrados pelo acidente. Os símbolos em preto indicam os pontos onde se adquiriram dados, a variação do campo magnético é da ordem de $500 \mathrm{nT}$. Em vermelho foi indicado a posição para se começarem as escavações (GALLI; CAMPOS; SOUSA, 2013).

\section{Objetivos e Justificativa}

Tendo em vista as limitações de acessibilidade em algumas campanhas de magnetometria terrestre, além do alto custo de aerolevantamentos convencionais, o principal objetivo deste trabalho foi avaliar o potencial do uso de Veículos Aéreos Não Tripulados (VANTs), na magnetometria e comparar esta técnica com a técnica terrestre exemplificada. Para tanto, foi necessário desenvolver alguns objetivos específicos:

I)

Prototipar um magnetômetro leve e de baixo custo a partir de sensores microeletromecanicos (MEMS).

II) Detectar anomalias magnéticas de uma área com alvos conhecidos embarcando esse protótipo de magnetômetro em um VANT de pequeno porte (menos de $25 \mathrm{kgf}$ ).

III) Comparar a qualidade dos dados coletados e os custos da técnica magnetométrica aerotransportada por VANT, com a técnica terrestre usada na pedreira em 2011.

\section{Material e Métodos}

A partir de sensores MEMS de leitura de campo magnético do tipo Fluxgate disponíveis no mercado, foi criado um sistema para ler e gravar esses dados ao longo do tempo (data-logger), a partir de um sistema Arduino, sensor GPS, conversor analógico-digital (ADC) de 16 bits, baterias de lítio e outros componentes eletrônicos. Mesmo o VANT possuindo vários desses componentes como baterias e GPS, o sistema magnetometro foi criado para funcionar completamente independente do drone, tendo este apenas a função de transporta-lo. O sistema data-logger foi organizado e embarcado em caixa resistente para transporte, e totalizou massa de aproximadamente 600 gramas.

Tendo em vista que o data-logger e o drone são equipamentos independentes, o acoplamento entre eles não necessita de fios e cabos de energia ou comunicação. Dessa forma, o acoplamento foi feito com auxílio de fio de nylon, conforme pode ser visualizado na figura 3 . A distância existente entre o data-logger e o drone visa minimizar o ruído magnético que o drone gera em cima do data-logger.

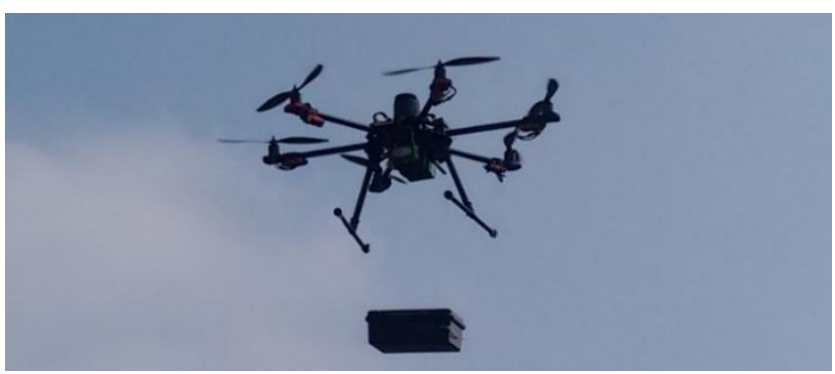

Figura 3 - Acoplamento do data-logger ao drone, com auxílio de fio de nylon.

Sabendo-se que os sensores magnéticos têm uma faixa de medida de $100.000 \mathrm{nT}$, variando de $-50.000 \mathrm{nT}$ a $50.000 \mathrm{nT}$, e que há $2^{16}$ valores possíveis que podem ser fornecidos pelo $A D C$, a resolução teórica máxima do sistema foi de $1,5258 \mathrm{nT}$.

Para o experimento, foi selecionada uma área (em São Paulo, dentro do campus da USP) onde se estacionou um carro próximo ao centro para este servir de fonte magnética, com o objetivo de fazer um estudo comparativo ao problema na pedreira para se encontrar os veículos soterrados. Todo o voo foi realizado de forma autônoma em 9 minutos, com altura aproximada de 3 metros em relação ao solo, e 6 metros de espaçamento entre as linhas de voo. A resolução dos dados, levando em conta os ruídos da eletrônica do sistema, da proximidade com o drone e todos os movimentos durante o voo foi da ordem de $10 \mathrm{nT}$. Não foi utilizado magnetômetro base devido ao fato do tempo de aquisição durar menos de 10 minutos e a variação diurna que se poderia obter nesse intervalo ser inferior a resolução do sistema $(\sim 10 \mathrm{nT})$. Foi constatado posteriormente a data do voo, 18/06/2016, que não houve tempestade magnética durante a aquisição dos dados.

\section{Resultados}

Os dados obtidos no voo foram interpolados e trabalhados através do software Oasis Montaj, que gerou um mapa de Campo Magnético Total (CMT) e um perfil $A B$ que atravessa a principal anomalia magnética, assim como um mapa de Amplitude do Sinal Analítico (ASA) para delimitar os limites laterais da fonte magnética. Os mapas e perfil podem ser verificados na figura 4. 

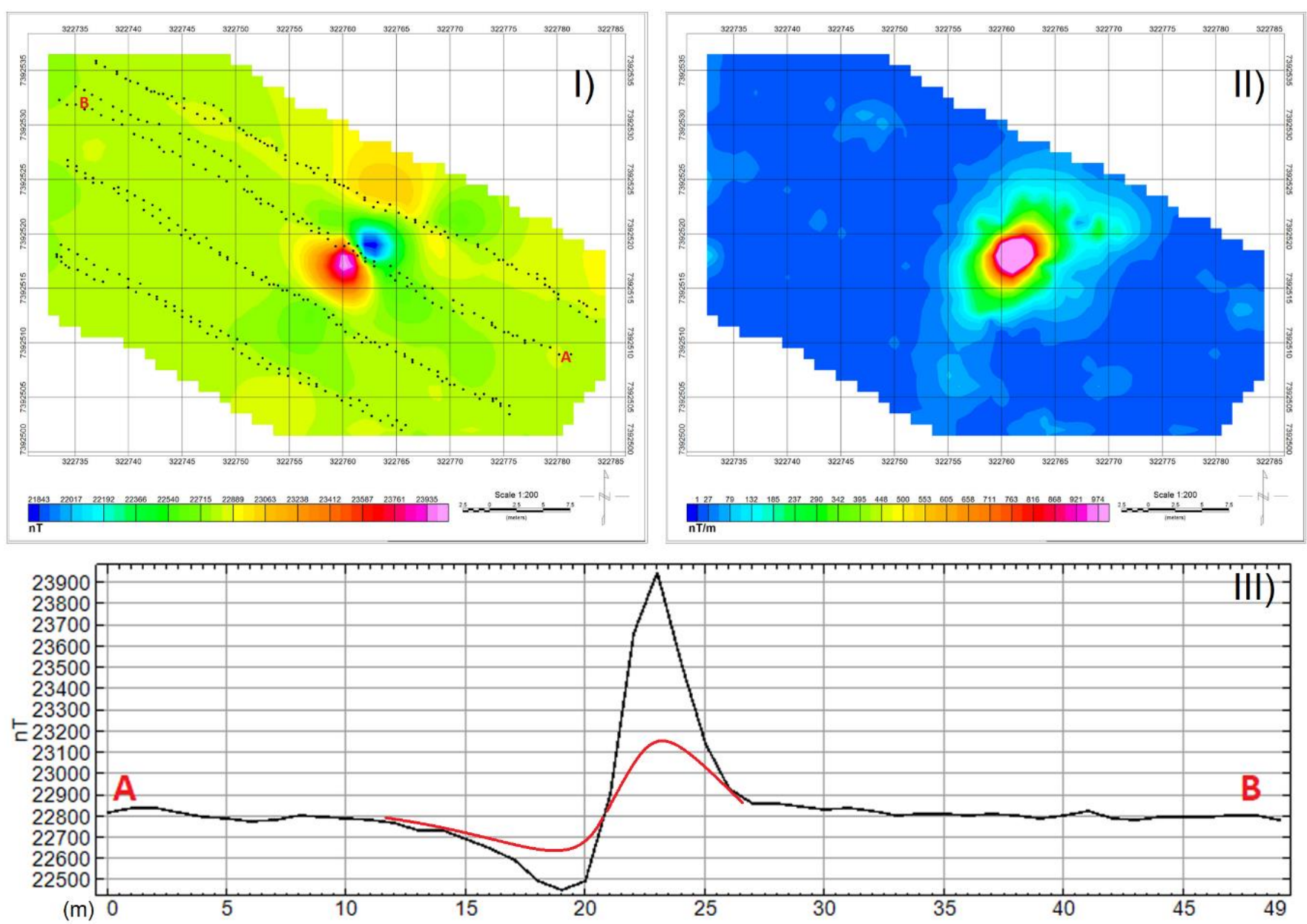

Figura 4 - I) Mapa de campo magnético total, obtido a partir de aerolevantamento realizado por VANT. Ao centro da área sobrevoada havia um automóvel orientado perpendicularmente à direção de voo. O processamento envolveu a interpolação dos dados por mínima curvatura com espaçamento de 1 metro. Os pontos pretos indicam os pontos de aquisição de cada dado. Devido um erro na programação do voo, os dados de ida e volta foram tomados na mesma linha. II) Mapa da amplitude do sinal analítico gerado a partir do mapa do campo magnético total, a anomalia magnética assim como seus limites laterais ficam mais evidentes. III) Perfil $A B$ do mapa de campo magnético total (em preto), a anomalia tem amplitude de aproximadamente $1500 \mathrm{nT}$, em vermelho foi feita uma curva de aproximadamente $500 \mathrm{nT}$ para evidenciar que a anomalia do problema da pedreira (figura 2) também poderia ser percebida pelo magnetômetro aerotransportado mesmo com resolução da ordem de $10 \mathrm{nT}$.

\section{Discussão}

Por uma questão de segurança, considerou-se que a autonomia do drone utilizado nesse estudo foi de 10 minutos de voo com carga (data-logger) de aproximadamente 600 gramas. Desta forma, obtendo uma medida a cada 5 metros (velocidade de $18 \mathrm{~km} / \mathrm{h}$ ), é possível mapear $3 \mathrm{~km}$ lineares de magnetometria em 10 minutos, depois disso é necessário trocar as baterias antes de se lançar em um novo voo. Em regiões de difícil acesso, não é comum uma equipe de magnetometria terrestre produzir muito mais do que $4 \mathrm{~km}$ lineares por dia, e o custo do $\mathrm{km}$ linear custa na maioria dos casos entre $R \$ 200,00$ e $R \$ 600,00$, devido a questões especificas de cada área de estudo, sem considerar o custo para abertura de picada, que também pode variar bastante e ficar muito elevado em áreas de difícil acesso e com terreno acidentado.

Todos os componentes que constituem o sistema datalogger a partir de sensores fluxgate custaram, em junho de 2016, aproximadamente $R \$ 3.000,00$. O drone utilizado teve seus componentes eletrônicos estimados em $R \$ 10.000,00$. Para operação em campo não é necessário mais do que uma pessoa treinada.

\section{Conclusões}

Com o objetivo de estudar anomalias magnéticas com mais de $100 \mathrm{nT}$ de amplitude, a técnica aerotransportada por VANT será mais vantajosa, por facilidades durante a 
logística, menor custo e redução do tempo de aquisição dos dados.

O acidente que ocorreu na pedreira em Santos em 2011, foi um caso em que era perigoso demais para se realizar magnetometria terrestre e a área era pequena demais para se contratar um serviço de magnetometria aerotransportada. A área em questão era menor que 1 hectare e devido à amplitude da anomalia magnética aproximadamente $500 \mathrm{nT}$ ), a magnetometria por VANT seria apropriada e não ofereceria risco para as pessoas.

Os VANTs se mostraram uma ferramenta promissora para a geofísica aplicada, uma vez que foi possível criar um data-logger com uma boa relação entre peso, custo financeiro e resolução de dados.

Nos últimos anos os VANTs evoluíram bastante em sua automação, estabilidade, capacidade de carga e tempo de voo. Atualmente, já existem drones com capacidade de carga superior a $2 \mathrm{kgf}$ e autonomia de voo de mais de 45 minutos. Em alguns anos essas tecnologias se tornarão cada vez mais acessíveis e vão representar mudanças significativas na forma que dados geofísicos são adquiridos.

\section{Agradecimentos}

Agradecimentos à Universidade de São Paulo, aos professores Wladimir Shukowsky, Eder Molina e Carlos Alberto Mendonça do Instituto de Astronomia, Geofísica e Ciências Atmosféricas e também à Otávio Gandolfo e Vicente Galli do Instituto de Pesquisas Tecnológicas.

\section{Referências}

GALLI, V. L.; CAMPOS, G. C. DE; SOUSA, L. G. DE. Ensaio geofísico de magnetometria aplicado na localização de equipamento soterrado. 13th International Congress of the Brazilian Geophysical Society. Anais. Rio de Janeiro: Sociedade Brasileira de Geofísica, 2013

KEAREY, P.; BROOKS, M.; HILL, I. Levantamento magnético. In: Geofísica de exploração. 1. ed. São Paulo: Oficina de textos, 2009. p. 438.

MARANGONI, Y. R. O campo magnético - conceitos e aplicações em geofísica: 3. São Paulo, 2014. 